\title{
ARTÍCULOS
}

\section{"MI HIJO ES AUTISTA»: NORMALIDAD Y MATERNIDADES DIFERENTES. UNA APROXIMACIÓN ANTROPOLÓGICA A LAS DIMENSIONES SOCIALES DEL AUTISMO}

"MY SON IS AUTISTIC": NORMALITY AND DIFFERENT MATERNITIES. AN ANTHROPOLOGICAL APPROACH TO THE SOCIAL DIMENSIONS OF AUTISM

\author{
Angélica Gutiérrez-González ${ }^{1}$ \\ Asociación Autismo Sevilla \\ Esteban Ruiz-Ballesteros ${ }^{2}$ \\ Universidad Pablo de Olavide
}

Recibido: 4 de mayo de 2019; Aprobado: 10 de diciembre de 2019

Cómo citar este artículo / Citation: Gutiérrez-González, Angélica y Esteban Ruiz-Ballesteros. 2020. «"Mi hijo es autista": normalidad y maternidades diferentes. Una aproximación antropológica a las dimensiones sociales del autismo». Disparidades. R evista de Antropología 75(2): e019. doi: <https://doi.org/10.3989/dra.2020.019>.

RESUMEN: En este artículo se presenta una aproximación antropológica a las experiencias de madres de personas con autismo. Dichas madres viven el ejercicio normativo de su maternidad en permanente tensión habida cuenta de la caracterización que se hace de la discapacidad de sus hijos e hijas desde el ámbito médico. La convergencia entre maternidad y autismo, considerados como fenómenos culturales, sujetos a conceptos y prácticas plurales, nos permitirá profundizar en cómo se resuelve esa tensión inherente al encuentro entre normalidad y discapacidad. Este es un contexto analítico privilegiado para comprender cómo se construye la diferencia, cómo se reproduce en el imaginario colectivo y de qué manera se desarrollan los procesos de integración y normalización de la discapacidad en la sociedad. Asimismo, a través de las trayectorias vitales de estas madres se evidencia la existencia de un discurso/práctica normalizador en torno al ser madre, construido en el marco de un contexto histórico, económico y social. Esta perspectiva analítica nos ayudará a mejorar el conocimiento sobre nuestros procesos de construcción de sentido en torno a la discapacidad.

PALABRAS CLAVE: Autismo; Maternidades; Etnografía; Normalidad; Discapacidad.

ABSTRACT: This article presents an anthropological approach to the experiences of mothers of people with autism. These mothers experience the normative exercise of their maternity in permanent tension given the way the disability of their sons and daughters is characterized in the medical field. The convergence between maternity and autism, considered as cultural phenomena, subject

1 Correo electrónico: angelicagutierrez@autismosevilla.org. ORCID iD: <https://orcid.org/0000-0002-0072-1241>.

2 Correo electrónico: eruibal@upo.es. ORCID iD: <https://orcid.org/0000-0002-6270-0593>. 
to plural concepts and practices, will allow a deeper exploration of how the tension inherent in the encounter between normality and disability is resolved. It is a privileged analytical context to understand how difference is constructed, how it is reproduced in the collective imaginary and how the processes of integration and normalization of disability in society are developed. Moreover, the life pathways of these mothers shed light on the existence of a normalizing discourse/practice regarding motherhood, built in a historical, economic and social context. This analytical perspective will help further knowledge about our processes of constructing meaning around disability.

KEYWORDS: Autism; Motherhood; Ethnography; Normality; Disability.

Copyright: (C) 2020 CSIC. Este es un artículo de acceso abierto distribuido bajo los términos de la licencia de uso y distribución Creative Commons Reconocimiento 4.0 Internacional (CC BY 4.0).

El diagnóstico te cae como una losa. Fue bestial, porque además yo tenía muy poca información. Yo había oído hablar del autismo: el niño mueble, el niño que no se relaciona, el niño que no sonríe, el niño que no es afectivo... Y mi hijo no encajaba en nada de eso, en el concepto que yo tenía del autismo, que era lo que decían los manuales. En esa época no había internet ni nada y lo que se podía leer era en un manual de psicología, y mi hijo no encajaba dentro de esos parámetros: se relacionaba con nosotros, era afectuoso, se reía y compartía cosas, a su manera (Manoli, madre de un chico con autismo de 35 años).

\section{INTRODUCCIÓN}

La discapacidad entendida como campo (Bourdieu 2003; Torres 2004), es un contexto privilegiado para reflexionar sobre la normalidad, su producción y reproducción. Discapacidad y normalidad son categorías que se definen recíprocamente de forma coyuntural e histórica (Alvarado, Andrés y González 2007; Rosato y Angelino 2009; Cisneros 2010). La línea imaginaria que las separa ha estado tradicionalmente vinculada a la ciencia médica y a la consideración patológica de determinadas características (Canguilhem 1970 De Miguel 1980; Menéndez 1990, 2018; Comelles y Martínez 1993), pero nada de todo ello puede comprenderse cabalmente si no se despliega una perspectiva cultural.

El reconocimiento personal y familiar de la discapacidad conlleva un duelo (Rosato y Angelino 2009; Osa 2018) ante la evidencia de verse situado más allá de la norma, desafiando la homogeneidad. Pero tam- bién implica aceptar formar parte de ese cajón de sastre que conforma lo diverso. Se genera así una tensión permanente, puesta de manifiesto no solo en las distintas etapas del ciclo vital, sino también en los hitos de la experiencia humana, como por ejemplo la maternidad y la crianza. Esa tensión tiene un gran valor analítico pues nos sitúa en el espacio liminar en que confluyen normalidad y diferencia.

La maternidad es una experiencia transformadora cargada de significados sociales y que trasciende la biología (Esteban 2000; Fernández 2014). Más allá de los intentos de la lógica patriarcal por homogeneizarla y reducirla a una cuestión individual, la maternidad es una experiencia humana clave para comprender la discriminación y la reproducción del poder en nuestra sociedad. Como experiencia humana, una de sus características definitorias es su diversidad.

En este artículo presentamos una aproximación antropológica a las experiencias de madres de personas con autismo que, como tales, viven el ejercicio normativo de la maternidad en permanente tensión, propiciando maternidades intensas o maternidades diferentes $^{3}$ (Bell 2004; Torres 2004; Ennis 2014) profundamente condicionadas por la explicación y caracterización que se hace de la discapacidad de sus hijos e hijas desde el ámbito médico.

El autismo es un fenómeno social que conforma un campo emergente para la investigación antropológica. Desde Eugen Bleuer, Grunya Sukhareva (Manouilenko y Bejerot 2015), Leo Kanner y Hans Asperger (Rivera 2004; García et al. 2006; Ramos 2010) el autismo ha tenido significados diversos, pasando de considerarse una forma de psicosis o esquizofrenia infantil, para luego incorporar visiones psicoanalíticas, o centrarse, como ocurre en la actualidad, en las causas genéticas del trastorno (Asenjo 2011). Los enfoques educativos, psiquiátricos y de diagnóstico han cambiado profundamente a través de los años (Kim 2012), si bien hay algunas características que se han mantenido como definitorias a través del tiempo: dificultades en las interacciones sociales, déficit en las habilidades comunicativas y un repertorio de conductas restringidas o inusuales (Dyches et al. 2004: 211). Considerado en la actualidad un trastorno del desarrollo, el autismo se

3 La experiencia de madres de personas con autismo muestra valores y conceptualizaciones propios de la maternidad normativa. Se hace uso del término «diferentes» o «intensas» para establecer la frontera entre la maternidad normativa y la que no lo es, la que se sitúa en los márgenes. 
define por una serie de criterios diagnósticos que incluyen alteraciones significativas en las habilidades socio-emocionales, en la comunicación social y el lenguaje, y la aparición de patrones restrictivos y repetitivos de comportamiento (APA 2013).

En general, la atención científica al autismo - preocupada por dilucidar sus posibles causas como medio para diseñar su tratamiento- ha considerado de escasa o nula relevancia su dimensión social y cultural (Daley 2002; Asenjo 2011), que por tanto, han sido excluidas del diagnóstico y la comprensión del fenómeno. De esta forma, el autismo se ha conceptualizado como un asunto estrictamente individual, una forma de diferencia originada fuera del ámbito de lo social (Grinker 2010: 177). Sin embargo, el autismo no puede ser entendido apropiadamente fuera de la cultura (Grinker 2008: 11). Desde esta nueva perspectiva deja de ser un trastorno para considerarse como un hecho social, conformado por significados que son compartidos y que se reproducen de manera colectiva; por tanto, con una evidente dimensión socio-cultural.

La convergencia de maternidad y autismo, considerados como dos fenómenos culturales, y por tanto sujetos a conceptualizaciones y prácticas plurales, nos permitirá profundizar en cómo se resuelve esa tensión inherente al encuentro entre normalidad y discapacidad, aquí ejemplificada en la tensión de la maternidad en torno a la persona con autismo. Mantenemos que este nudo tensional es uno de los contextos analíticos clave para comprender de manera eficaz cómo se construye la diferencia en el campo de la discapacidad, cómo esta se reproduce en el imaginario colectivo y de qué manera se llevan a cabo los procesos de integración y normalización de la discapacidad en nuestra sociedad. Asimismo, esta perspectiva analítica nos ayudará a mejorar el conocimiento de nuestros procesos de construcción de sentido en torno a la discapacidad.

La discapacidad atraviesa las experiencias vitales de la persona que porta esa etiqueta, para la cual se construye a nivel social una identidad en negativo, basada en la carencia de normalidad (Ferreira 2007; Rosato y Angelino 2009). Esta identidad deteriorada (Goffman 2012) impregna a la persona diagnosticada con autismo y a todo su entorno, trascendiendo al individuo y extendiéndose hacia su contexto social (Beckett 2015). De este modo, la etiqueta sirve para distinguir a unas familias de otras en tanto en cuanto funciona como engranaje entre capacitados y no capacitados (Araya 2007: 7). Como consecuencia, emergen discursos inculpatorios en el entorno médico y familiar (Waltz 2015) al tiempo que se activan respuestas resilientes en el ámbito relacional comunitario en forma de movimientos sociales. $Y$ es que la existencia de la discapacidad supone un gran desafío a la ideología de la normalidad/normatividad que está vigente en el imaginario colectivo, y que pretende homogeneizar discursos y prácticas sociales.

En este artículo focalizamos en el ejercicio de la maternidad de madres de autistas que han alcanzado la edad adulta, y que por tanto tienen una experiencia dilatada tanto de esa maternidad diferente como de la discapacidad en sí. Sus trayectorias vitales evidencian la existencia de un discurso/práctica normalizador o ideal en torno al ser madre, construido en el marco de un contexto histórico, económico y social determinado. Pero, al mismo tiempo, explicita la vigencia de un constructo análogo en torno a la idea de persona, como sujeto de derechos y obligaciones, según el contenido forjado en la modernidad, que colisiona con la condición de autista. Por eso es necesario encarar la normalidad y lo que significa alinearse fuera de ella, construirse al margen de la misma, con las consiguientes repercusiones a nivel social.

Desde un punto de vista teórico, estas páginas contribuyen de forma general al debate sobre la normalidad y la diversidad a través del análisis de maternidades diferentes en torno al autismo. Los espacios de tensión que suponen estos contextos de maternidad/autismo nos permitirán constatar imaginarios sobre ambos fenómenos, evidenciando que la práctica de la maternidad en situaciones de diversidad nos permite aproximarnos a las dimensiones sociales y culturales del autismo, cuestiones inexploradas hasta el momento desde una perspectiva antropológica. Esa tensión entre maternidad y autismo, pone de manifiesto una notable fractura entre ideales, expectativas y juicios morales, que ha provocado una respuesta colectiva en forma de movimiento social. Dicha movilización en torno al autismo, parece ser la consecuencia del discurso inculpatorio respecto a las madres, dominante en la ciencia médica durante largo tiempo.

Este artículo recoge parte de los resultados de una investigación más amplia que propone una aproximación al autismo desde dentro ${ }^{4}$, analizando entre otros

4 Se hace aquí un guiño doble, por una parte a la capacidad de la Antropología para analizar la cotidianidad y aplicar 
aspectos las experiencias que conllevan la maternidad y crianza de niños y niñas autistas. Nuestra etnografía del autismo incluye el análisis en profundidad de quince casos de madres de personas adultas con autismo en la ciudad de Sevilla, las cuales han compartido sus experiencias de maternidad atravesadas por diagnósticos médicos, intervenciones terapéuticas, estigmas, discursos de culpa materna y la emergencia de conceptos que ya creíamos parte del pasado. Pero también por pequeños grandes logros, la búsqueda incansable de respuestas y su articulación como movimiento social organizado, el apoyo de profesionales y la comprensión de otras madres con un objetivo claro y compartido: la felicidad de sus hijos e hijas.

\section{METODOLOGÍA}

Para aproximarnos a esta articulación entre discapacidad y maternidades, asumimos la «mirada antropológica» (Jociles 1999: 1), entendiendo que la etnografía explota la capacidad de aprender a mirar la otredad desde escenarios de cotidianidad (Ruiz Ballesteros 2013). Retomando el prefacio de Frazer a la obra de Malinowski, se trataría de "ver en relieve» (Malinowski 1986: 6) a los agentes en relación con el autismo. Del mismo modo lo entiende Olga Solomon, cuando recomienda "la comprensión del autismo como una experiencia personal, familiar y comunitaria, a través del análisis de las interacciones sociales, narrativas y la participación en actividades del hogar y en otros marcos institucionales: contextos educativos, servicios sociales y sanitarios ${ }^{5}$ » (Solomon 2010: 242).

La etnografía se ha llevado a cabo partiendo de estudios de caso, una estrategia de conocimiento que enraíza en el contexto y que da prioridad a la experiencia frente al conocimiento que busca leyes generales. Se trata de centrar la atención en las pequeñas cosas, o en palabras de Rorty «ceñirse a lo concreto

su mirada a fenómenos complejos como el autismo, y por otra parte a un libro de gran trascendencia en el ámbito asociativo dirigido a padres, madres y profesionales, publicado en 2012 y titulado El autismo desde dentro: una guía, cuya autora es Hilde De Clercq.

5 «Autism as a personal, family, and community/social group experience as evidenced through the analyses of social interactions, narrative accounts, and participation and engagement in activities in the home and educational, clinical, and other institutional settings» (Solomon 2010: 242). para re-encantar el mundo" (Flyvbjerg 2004: 53). A pesar de las críticas, se asume aquí la capacidad de generalización a través de casos si bien se reconoce también que «la generalización formal está sobrevalorada como fuente de desarrollo científico, mientras la fuerza del ejemplo está subestimada» (Flyvbjerg 2004: 44). Mediante el estudio de caso etnográfico nos acercamos a la vida real y comprobamos en ella cómo se expresan de forma tangible los fenómenos que nos interesan.

Los casos analizados han tenido como lugar común una asociación de familias de personas con autismo, en la que desarrolla su actividad profesional como trabajadora social una de las autoras. Dicha organización se dedica desde su constitución en 1978 a la atención y provisión de servicios específicos para las personas con autismo y sus familias, siendo la entidad de referencia respecto al autismo en la provincia de Sevilla.

Para el estudio que sirve de base a este artículo, se seleccionaron 15 casos de personas con autismo de edad adulta, que han sido analizados desde tres perspectivas: el de las propias personas con autismo, el de sus familias y el de los profesionales de servicios de apoyo. La selección específica de los casos ha estado orientada por los profesionales de la asociación que trabajan directamente con ellos, y que conocen sus trayectorias y necesidades de apoyo, de manera que todos los protagonistas tienen o han tenido contacto y vinculación con la organización en algún momento de su vida. No obstante, de manera más específica, para los objetivos de este artículo nos centramos en el punto de vista de las familias, el cual ha sido recogido a través de observaciones y entrevistas en profundidad desarrolladas durante tres años (2015-2018).

Las observaciones se han desarrollado en contextos formales como asambleas y reuniones de familias, actividades formativas y tutorías con profesionales. También en contextos informales: domicilios, encuentros casuales no programados y charlas ocasionales en actos sociales o trayectos de viajes.

Las entrevistas en profundidad se han llevado a cabo en la entidad, acordando previamente la cita de manera programada por ambas partes y asegurando un entorno apropiado. Para favorecer la sistematización de las mismas se elaboró un guion de entrevista que incluía en primer lugar datos generales de carácter identificativo (nombre, edad, estado civil, 
ocupación). En segundo lugar, se explicaba el objetivo del estudio y una pequeña contextualización, para pasar en tercer lugar a recorrer las diferentes trayectorias siguiendo determinados hitos vitales: la formación de las familias, el nacimiento de hijos e hijas, la etapa infantil, el proceso diagnóstico, la asistencia a servicios de salud mental, la etapa escolar desde la educación infantil hasta la primaria y secundaria, la adolescencia, la relación con los profesionales de apoyo y sus motivaciones para aproximarse al movimiento asociativo, y la etapa adulta, orientada fundamentalmente a procesos de autodeterminación y vida independiente que ya se han producido o que se encuentran en proceso.

El trabajo de campo con familias contemplaba inicialmente a todos los miembros de la unidad familiar, si bien con el transcurso de las diferentes citas, los casos particulares de las madres cobraban cada vez mayor protagonismo. Eran ellas las cuidadoras principales; aunque podían recibir apoyos a nivel interno o externo de la familia, resultaron ser las madres las que estaban disponibles para las entrevistas y retenían en su memoria hasta el más pequeño detalle de las trayectorias de sus hijos e hijas. Por esta cuestión, el trabajo de campo respecto a las familias ha acabado consistiendo en la recopilación de las experiencias de las madres de las personas con autismo. Estas mujeres tienen en común ser mayores de cuarenta y cinco años y tener hijos e hijas mayores de edad entre dieciocho y cincuenta y dos años- con diagnóstico de autismo. Viven mayoritariamente en la ciudad de Sevilla, si bien algunas residen en la zona metropolitana, y todas gozan de un nivel socioeconómico medio, siendo muchas de ellas trabajadoras por cuenta ajena en profesiones liberales. Otras se encuentran ya disfrutando de su jubilación. Estas personas son o han sido beneficiarias de algún servicio prestado por la asociación, de la que son miembros activos.

\section{LA DISCAPACIDAD COMO CUESTIÓN SOCIAL}

La discapacidad es un ámbito de investigación tradicionalmente olvidado por las ciencias sociales y que sin embargo está presente en todas las culturas (Ferreira y Ferrante 2011; Guerrero 2011; Gómez 2014). En cada una de ellas, las percepciones y explicaciones sobre esta condición anómala han variado, pasando de tener causas mágicas o religiosas, a ser resultado de la genética, el ambiente, o una combinación de ambas (Aguado 1995; Seoane 2011). En este sentido se reconocen una serie de modelos (Verdugo 2003; Palacios y Romañach 2008; Palacios 2008; Villa y Arnau 2008) para clasificar las explicaciones en torno a la discapacidad, que no cuentan con un carácter de progresión, sino que confluyen y comparten espacio en lo social:

\section{- Modelo de prescindencia \\ - Modelo médico-rehabilitador \\ - Modelo social}

En el modelo de prescindencia, se considera que "las causas de la discapacidad tienen un motivo religioso" y que "las personas con discapacidad son innecesarias», por lo cual la actuación de la sociedad será «a través de políticas eugenésicas o bien situándolas en el espacio destinado para anormales y clases pobres» (Palacios 2008: 26). Se trata de un modelo que se asienta en una perspectiva biologicista (Villa y Arnau 2008) que centra la discapacidad en la persona.

Para el modelo médico-rehabilitador, «las causas de la discapacidad son científicas» y el "fin último será la normalización del individuo» (Palacios 2008: 26), ya que se ha entendido la discapacidad como «un tema médico y un problema individual» (Oliver 1998: 34), lo cual viene también a encuadrarse en un momento en el que la ciencia se presenta como un discurso central y globalizador que se erige como sacro social, lo que Comelles (2007: 10) denomina la "hegemonía del fundamentalismo biológico», y todo esto al mismo tiempo que se está conformando la noción de normalidad (Corbett 1997; Foucault 2000, 2004; Rodríguez y Ferreira 2010; Hollin 2014). Ya advierte Foucault (2000: 155) que la ciencia médica, en concreto la psiquiatría

introduce la norma, entendida como regla de conducta, como principio de conformidad, a la que se oponen la irregularidad, el desorden, la extravagancia, la excentricidad [...]: lo normal, al que se opondrá lo patológico, lo mórbido, lo desorganizado y el disfuncionamiento.

Es precisamente por la influencia de Foucault (Oliver 1998) por lo que la ciencia social se aproxima a la discapacidad desde el funcionalismo de Parsons y el "papel del enfermo en el contexto social», y desde el interaccionismo simbólico, que analiza aquella como desviación de la norma. Es aquí procedente comentar las aportaciones de Goffman, que impulsa la perspectiva interaccionista «mediante el uso del concepto de estigma» (Oliver 1998: 38). En 1963 
Goffman publica su obra Estigma: la identidad deteriorada, en la que hace referencia a varias cuestiones relevantes para este artículo: por una parte, la información que producen los sujetos estigmatizados sobre ellos mismos en la interacción con los otros, y por otra parte, la percepción del estigma no en el individuo sino en la relación social en la que unos individuos etiquetan a otros en función de ciertos atributos.

Por último, el modelo social de discapacidad alude a una cuestión básica para este artículo, que es la construcción social de la discapacidad (Verdugo 2003; Bagatell 2010; Solomon 2010). En este sentido se asume que solo a través de la integración de las diferentes perspectivas (médicas, psicológicas, sociales y culturales) podemos llegar a una comprensión cierta de la discapacidad (Solomon 2010; Verdugo 2003).

La discapacidad es, por tanto, un elemento que estructura la identidad de la persona y de su entorno, sobre la base de la ideología de la normalidad/normatividad (Foucault 2000; Araya 2007; Rosato y Angelino 2009; Gómez 2014). Canguilhem, por su parte, señala que lo normal es, por una parte, «aquello que es tal como debe de ser» y por otro lado, «aquello que se encuentra en la mayoría de los casos», lo que implica al mismo tiempo un hecho y un juicio de valor, este último «solo cobra sentido y cabe ser entendido por contraposición a lo no normal, a lo anormal» (Canguilhem 1970: 91). La construcción social de la realidad implica la asociación de la discapacidad con el par normal/anormal (Araya 2007) de manera que esta visión dicotómica favorece los procesos de inclusión o exclusión asociados a la experiencia social de la discapacidad. Desde este punto de vista, la discapacidad «indicaría dos realidades: la falta de ciertas capacidades que la mayoría de las personas poseen, y los discursos y las prácticas institucionales que sancionan el orden normativo cumpliendo una función moral» (Rodríguez y Ferreira 2010: 76).

Es importante comprender que los significados asociados a las capacidades son arbitrarios, "ya que aluden a las estructuras socioculturales y económicas, resultantes de la evolución histórica y modelan las funciones propias del cuerpo en el tipo de sociedades de las que somos miembros» (Rodríguez y Ferreira 2010: 76). La discapacidad tiene más que ver con la idea colectiva de normalidad, su concepción histórica y relacional, que con las personas con capacidades diversas. Las personas con discapacidad son aquellas que «en virtud de ciertas peculiaridades de su cons- titución biológica, encuentran dificultades para su desenvolvimiento cotidiano, en comparación con las personas que no poseen esas peculiaridades» (Rodríguez y Ferreira 2010: 67). Estas personas se desenvuelven en un entorno en el que hay personas sin discapacidades, lo cual, tal como señala Ferreira (2007) tiene varias implicaciones. En primer lugar, que su existencia y sus prácticas están dominadas por su condición. En nuestro caso de estudio, dejan de ser personas para pasar a ser autistas. Ya no importan sus expectativas, sus intereses o deseos. Prácticamente todo se reduce a la experiencia de ser autista, lo que implica no tener ni las mismas habilidades que otros, ni aspiraciones ni tampoco derechos. Se relegan de este modo las características positivas y las fortalezas con las que cuenta todo ser humano para centrarse en lo que falta, en lo que no se tiene: normalidad.

En segundo lugar, provoca la tendencia a su homogeneización: personas que tienen dificultades para la comunicación, para la interacción social y la comprensión de los otros, olvidando que estas son características genéricas y que pueden compartirse en mayor o menor medida. La heterogeneidad forma parte de la especie humana, también de las personas con autismo. Esta representación de los autistas los sitúa en oposición a los no discapacitados, «infiriendo una identidad en negativo» (Ferreira 2007: 1), es decir, enfatizando esa carencia de normalidad e invisibilizando otros valores personales que son percibidos de manera positiva, pero solo en personas sin autismo. Ferreira (2007: 6) indica que:

\footnotetext{
El discapacitado se da cuenta de su diferencia, no la construye. La diferencia es definida por el otro $y$, a su vez, la identidad que de ella surge no es una según la cual la discapacidad constituya un hecho propio diferenciador, sino la ausencia de rasgos identitarios respecto al otro. Es una identidad heterónoma y en negativo; es una identidad excluyente y marginalizadora. Es una no-identidad. Es la identidad de la insuficiencia, la carencia y la falta de autonomía.
}

En este sentido, resurge de nuevo la idea de normalidad, teniendo en cuenta que la identidad social de las personas con discapacidad se construye sobre la base de la anormalidad, de la carencia. Esto genera exclusión y opresión, al tiempo que discriminación y desigualdad (Ferreira 2007). Todo ello tiene, en tercer lugar, consecuencias directas a nivel relacional (Allué 2002; Ferreira y Ferrante 2011), en el reconocimiento de sus derechos y en las oportunidades para 
ejercerlos, convirtiendo esta realidad ontológica en una cuestión social de especial importancia.

Por último, es imprescindible tener en cuenta que precisamente por su carácter de constructo, los discursos y prácticas asociados a la discapacidad «son creaciones culturales y por ende pueden ser modificadas» (Araya 2007: 9). Comprender la discapacidad supone entonces comprender su contexto, su historia, sus valores, sus discursos, sus lenguajes, sus normas, sus imágenes, y también sus contradicciones. Para que este ejercicio sea efectivo es imprescindible «la desnaturalización respecto al modo hegemónico en el que ha sido entendida» (Gómez 2014: 395), lo que por otra parte amplía las formas de ser y estar en el mundo.

\section{LA CONSTRUCCIÓN SOCIAL DE LAS MATERNIDADES}

Los discursos hegemónicos han hecho de la maternidad una cuestión individual, natural y exclusiva de las mujeres, ocultando sus dimensiones socio-histórica y cultural; forjando, por tanto, una lectura etnocéntrica y naturalista del hecho de ser madre (Esteban 2000). Las ciencias sociales tampoco han considerado la maternidad como objeto prioritario de estudio, evidenciando «el poder de la ciencia en la generación y perpetuación de la subordinación de las mujeres» (Fernández 2014: 23). A pesar de todo ello, evidentemente, la maternidad es un fenómeno que va más allá del hecho biológico. Dirigir la mirada hacia estas madres de personas autistas y a sus experiencias vividas desde una perspectiva antropológica, implica analizar críticamente la ideología cultural y los procesos históricos bajo los cuales se construyen discursos y prácticas en torno a la maternidad (Bell 2004).

Es a partir del siglo XVIII, con los antecedentes de la Ilustración y la Revolución Francesa, cuando comienza a definirse el ideal de buena madre,

en el creciente contexto de separación de las esferas pública/privada, naturaleza/cultura, producción/reproducción y de diferenciación natural de los sexos, mediante las ofensivas moralizadoras de la nueva maternidad como eje identitario de la esencia femenina (Fernández 2014: 26).

En ese momento se define a las mujeres en función de su biología y la división sexual del trabajo pasa a imponer una serie de valores en función del género, los cuales «van a guiar las representaciones y prácti- cas socialmente aceptadas sobre el deber ser» (Torres 2004: 28). De este modo, la mujer madre se sitúa en el ámbito doméstico y de reproducción social y le son asignadas cualidades como «la abnegación, el sacrificio, virtudes como el ser para otros y habilidades como la ubicuidad; elementos que, sin ser intrínsecamente negativos, denotan situaciones de exclusión, subordinación y dominación al convertir a la mujer en dependiente y sumisa dentro de un orden social establecido» (Torres 2004: 28). Este discurso ideal mitifica la maternidad «instando a las mujeres a ejercer de buenas madres dadas sus virtudes naturales femeninas, entre las que se encuentra el instinto maternal» (Fernández 2014: 26).

En las sociedades capitalistas modernas (Ennis 2014) permanece este ideal de maternidad y junto a él la conceptualización de buena madre y su construcción opuesta, la mala madre. De esta manera y «por mandato cultural las mujeres deben ser madres sacrificadas, abnegadas, emotivas, entregadas y por supuesto, amorosas, todo ello según el modelo de socialización que se espera de las mujeres» (Araya 2007: 15), frente a mujeres profesionales y con carreras competitivas (Hays 1996). Tal como indica Torres (2004: 113) el modelo de madre ideal vigente incorpora nuevos significados: «La madre trabajadora, la madre profesional, la madre independiente [...] lo que define un panorama sumamente complejo». En este sentido, el referente de la maternidad no solo es exigente, sino en ocasiones inalcanzable, lo que trae consigo el sentimiento de culpa y la estigmatización de aquellos desempeños de la maternidad que se entienden como inadecuados o que no encajan en ese ideal (Ennis 2014).

La identidad de las mujeres se construye en gran parte en torno a la maternidad (Fernández 2014), asignándole una serie de valores como la paciencia, la entrega y el sacrificio (Torres 2004). Se produce entonces una «maternalización de las mujeres» (Imaz 2010: 145) que sustentada desde teorías psicológicas sobre el apego (Esteban 2000) desemboca en la centralidad absoluta de la relación madre-hijo. Por su carácter de constructo histórico, se sigue manteniendo ese ideal que define la base sobre la cual se estructura lo adecuado y lo inadecuado, lo que debe y no debe ser una madre, una especie de «maternidad moral» (Fernández 2014: 31) que está muy relacionada con la ubicación de las mujeres en la esfera doméstica y su realización personal a través del cuidado de terceros, ya sean descendientes o ascendien- 
tes, del que son las principales responsables (Fernández 2014; Kelly 2016; Gutiérrez y González 2017). Se asume que «las madres son las cuidadoras naturales de los hijos, por lo que cualquier transgresión de la norma es calificada negativamente al ser un elemento de inestabilidad» (Torres 2004: 121). La asignación de las tareas de cuidados se realiza bajo esos criterios morales que hacen que las mujeres madres asuman grandes cargas de trabajo "que además solo ellas pueden realizar de manera adecuada y que son su obligación, lo que favorece la aparición de sentimientos tales como la soledad, el resentimiento y el cansancio» (Araya 2007: 16).

Desde los años 70 del siglo XX se ha producido lo que Fernández (2014: 31) denomina una «re-idealización de la maternidad», una vuelta a la naturalización de la maternidad que no deja de ser un reflejo de su imagen más tradicional, que se contrapone con ideales como «la defensa de una mayor capacidad de decisión y libertad para las mujeres» (Fernández 2014: 33) que se desarrollan con intensidad en ese momento histórico. Así, proliferan estudios en los que se analizan otras maneras de comprender la maternidad (Bell 2004), si bien esta sigue siendo «una metáfora organizadora de la sociedad» (Fernández 2014: 34). Se trata entonces de experiencias que son diversas y particulares, y que deben distinguirse del modelo hegemónico dominante. Esta nueva perspectiva va a focalizar en el contexto estructural en el que la mujer es madre, así como en la pluralidad de identidades, significados y formas de relación con los hijos que se pueden dar en la maternidad (Bell 2004: 45). Para realizar un adecuado acercamiento a las maternidades particulares es imprescindible comprender cómo la maternidad está mediada por la cultura y los discursos en torno a la biomedicina (Bell 2004) al tiempo que se trata de desgranar los múltiples significados que se asocian a la maternidad (Ennis 2014). En este sentido, el reconocimiento de otras maneras de ejercer la maternidad implica un ejercicio político de visibilización de otras maternidades posibles (Torres 2004).

\section{AUTISMO Y MATERNIDADES DIFERENTES. UNA APROXIMACIÓN ETNOGRÁFICA}

Las maternidades, como hemos visto, trascienden el hecho biológico y se convierten en un fenómeno social que es construido recursivamente. En este sentido, nuestro objetivo es mostrar cómo las «madres diferentes» elaboran sus maternidades y cómo estas están atravesadas por conceptos afines a la discapacidad como campo, a la medicalización del autismo y por discursos hegemónicos asociados a la clínica. En este contexto, emergen «procesos terapéuticos, económicos, culturales, psicológicos que [...] se despliegan como limitaciones, carencias y negaciones» (Torres 2004: 36). Las madres deben asumir el modelo ideal impuesto y afrontar que sus maternidades son maternidades diferentes (Bell 2004; Torres 2004; Ennis 2014).

Estas maternidades diferentes generan una posición ambigua y conflictiva frente a los significados asociados a la maternidad ideal. Se trata de una maternidad intensa (Bell 2004) que se caracteriza porque su responsabilidad recae exclusivamente en la madre biológica, se compone de tareas de cuidado centradas en el niño y la niña, y las mismas se caracterizan por el sacrificio y la predisposición de las mujeres a cuidar de otros antes que de sí mismas. Esto muestra también cómo la maternidad es una cuestión emocional (Bell 2004: 68). Estas maternidades diversas se encuentran a su vez fuertemente modeladas por el contexto económico y político de las sociedades capitalistas modernas, en dos sentidos claramente diferenciados pero que se retroalimentan entre sí. Por una parte, respecto al consumo de productos y servicios que mejoran los pronósticos de la discapacidad en múltiples variantes: la disponibilidad de especialistas para el diagnóstico, la demanda de herramientas para la detección, la creación de servicios de apoyo y la puesta en marcha de investigaciones, todo ello configura lo que podemos denominar la industria del autismo. Y, por otra parte, al refuerzo del vínculo entre madre e hijo basado sustancialmente en las teorías del apego que consolidan la lógica de una sociedad patriarcal que insiste en que los cuidados y la reproducción social corren a cargo casi exclusivamente de las mujeres (Ennis 2014: 2). Esta ideología sobre la maternidad es fácilmente asumible en las sociedades occidentales, en las que se ha reforzado el ideal de buena madre en base a que sean las mujeres «las encargadas principales de los cuidados, las mejores conocedoras y garantes de su bienestar» (Fernández 2014: 31).

Del mismo modo, estas maternidades se encuentran fuertemente condicionadas por las explicaciones y construcciones discursivas sobre la discapacidad de sus hijos e hijas. Las madres de autistas navegan en un mundo profundamente medicalizado, en el que 
los profesionales médicos son reconocidos como portadores legítimos de los saberes expertos (Foucault 2000; Torres 2004), frente a los cuales se ven obligadas a asumir, inicialmente, un papel pasivo y naturalmente asociado a la provisión de cuidados.

Para aproximarnos al desarrollo de la maternidad de personas con autismo vamos a focalizar etnográficamente en un momento clave - el diagnóstico- y en una práctica estratégica -la intervención de los profesionales de salud mental-. Estos dos ámbitos desvelan las tensiones, circunstancias y cualidades más relevantes para comprender la experiencia de las madres de personas con autismo.

\subsection{Proceso diagnóstico}

El momento del diagnóstico viene normalmente precedido por una experiencia -la propia maternidad-que las madres describen como una de las más felices de sus vidas. En la mayoría de los casos se trata de madres primerizas para las cuales el hito de la maternidad ha sido profundamente deseado. Mujeres jóvenes y no tan jóvenes para las cuales la maternidad es una experiencia que va unida a su realización personal y para las cuales ser madre se ha convertido en una experiencia transformadora de sí mismas y de su realidad. Tras unos meses de adaptación y un ejercicio normalizado de la maternidad estas madres comienzan a percibir las primeras señales de alerta en sus hijos, que se evidencian con una regresión en el desarrollo normativo, una interrupción o ralentización de los procesos habituales de crecimiento y un cambio repentino en determinadas conductas, como la relación particular y poco frecuente con algunos objetos y con otras personas. Muchas de ellas describen cómo esas sospechas eran contrastadas por las maestras de educación infantil, que notaban algo diferente en los niños y niñas pero no sabían cómo explicarlo: tenían comportamientos diferentes en el recreo y no se relacionaban con sus compañeros como era esperable.

Su tutora me comentó que había algo distinto en él, notaba que en clase estaba muy distraído. En el recreo empezaba a correr y a dar vueltas, no se relacionaba con otros niños, hacia muchos ruiditos en clase. Entonces a raíz de ahí pues me dijeron que lo llevara a algún sitio a que lo vieran, a ver si tenía algún problema físico (Mar, madre de un chivo con autismo de 20 años).
Eran niños y niñas que se desarrollaban con normalidad, con interacciones adecuadas en su entorno, con lenguaje incipiente pero que de repente, en torno a los dos años de edad, en lugar de avanzar, comienzan a retroceder. Las señales de alarma se centran en la falta de interés por interactuar con otras personas, la pérdida de mirada a los ojos, la utilización poco convencional de juguetes y otros objetos y respuestas desmedidas por la imposibilidad de controlar la frustración.

Cuando tenía tres años empezaron a decirme en la guardería que tenía muchos berrinches, se tiraba al suelo, se mordía, se daba golpes, y eso era un problema. Le derivaron a servicios sociales, y empezaron a verlo en atención temprana dos veces en semana (Inma, madre de un chico con autismo de 24 años).

Ante estas señales, se abre un período de gran incertidumbre y preocupación en el que se producen numerosas preguntas que se trasladan a los profesionales de la medicina. Las inquietudes se agravan cuando se percibe en el entorno médico la falta de respuestas o de un tratamiento adecuado para sus hijos e hijas. Durante este proceso las familias experimentan una gran soledad y mucha desorientación, en parte por la falta de formación de los profesionales sanitarios, que en ocasiones no estaban seguros del camino que debían transitar. Desde análisis de sangre, radiografías y electroencefalogramas, las preguntas sin responder cada vez son más numerosas, y la incertidumbre crece. En ocasiones, la recomendación médica era simplemente esperar a que los síntomas se mitigaran o incluso llegaran a desaparecer por sí mismos, algo que aumentaba la angustia de las madres. Esto podía provocar en ocasiones enfrentamientos y graves tensiones con los médicos de atención primaria, y la búsqueda de alternativas en otros profesionales, fuera de los servicios públicos, los cuales no siempre se guiaban por criterios éticos. En otros casos, el diagnóstico directamente era erróneo, generalmente asociado a un problema auditivo que no era tal y se producía la derivación a otros especialistas, generalmente del tercer sector, que, sin embargo, parecían tener más experiencia:

\footnotetext{
La chica de la asociación de sordos me dijo: yo soy especialista en sordos, pero no en autismo, y tu hijo es autista (Carmen, madre de un chico con autismo de 19 años).
}

En este contexto, se genera una situación de gran vulnerabilidad que desencadena por parte de las ma- 
dres la construcción del duelo, el reconocimiento de la pérdida del hijo o hija que conocían y para el cual habían imaginado ya un futuro. Las expectativas como madre comienzan a caerse como un castillo de naipes, con la necesidad de empezar de nuevo. Y no es solo pérdida simbólica del hijo o hija, sino también la alteración familiar, al tiempo que aparece una gran tensión por conocer cómo van a ser sus vidas a partir de ese momento. Escuchar finalmente y tras este complejo proceso la palabra autismo mitiga, en ocasiones, esa situación de incertidumbre constante, pues parece que darle nombre a lo que ocurre es el punto de inflexión para comenzar a encarar y quizá resolver el problema.

Pues la verdad es que a mí no me pareció muy trágico. El comienzo, fue duro, pero... Cuando escuché lo del autismo, entonces no me chocó. Sabía que iba a tener que luchar mucho porque ya lo había escuchado, y lo hice desde el primer momento (Loli, madre de un chico con autismo de 39 años).

En otros casos, sin embargo, la palabra autismo evoca misterio, desconocimiento, temor, inseguridad y también imágenes y comportamientos que ha plasmado el cine con mayor o menor acierto a lo largo de los años. Pero sobre todo, una exigencia: la de cumplir, a pesar de todo, con las expectativas asociadas al ideal de madre.

\subsection{INTERVENCIÓN DESDE EL ÁMBITO DE SALUD MENTAL}

Sin duda, los datos más relevantes del trabajo de campo provienen de los testimonios sobre esta práctica estratégica en el ámbito de la salud mental. No obstante, es necesario antes realizar un breve recorrido histórico para contextualizar apropiadamente esta cuestión.

En los años 40 y 50 del siglo XX el autismo era definido desde el ámbito psiquiátrico como una psicosis extraña y aguda, que se caracteriza por problemas en el lenguaje y la interacción (Chamak 2008: 81). En un momento dominado por la corriente psicoanalítica, muchos profesionales situaban a las madres y su comportamiento en el centro del fenómeno del autismo. Se establecían así relaciones causales entre la conducta familiar, pero concretamente la de las madres, y la aparición del autismo (Chamak 2008). En estos casos se recomendaba como tratamiento para el autismo un cambio de contexto, que implica- ba en ocasiones la separación de la familia y la intervención psicológica individual con las madres. Bruno Bettelheim (2012), con su obra La fortaleza vacía fue quizá uno de los investigadores sobre el autismo que más proyección social tuvo en la época, también en el ámbito español. Seguramente tuviera cierta influencia el hecho de que hasta 1978 su libro fue el único traducido en España sobre esta temática ${ }^{6}$. En él realiza una comparación entre los síntomas de niños con autismo con los prisioneros de un campo de concentración nazi, experiencia que el autor padeció en primera persona en Buchenwald. Más concretamente establece un paralelismo entre el comportamiento de los guardias nazis de esos campos y el de las que llama «madres nevera ${ }^{7} »$ (refrigerator mothers) que no muestran afecto y prácticamente maltratan a sus hijos.

Si bien con el paso del tiempo, es acusado de numerosos delitos e incongruencias en sus investigaciones y olvidado por la disciplina que lo encumbró, las repercusiones de su publicación en la conformación del concepto de madre nevera y su afianzamiento en el imaginario colectivo han permeado trágicamente en la práctica médica hasta hace relativamente poco (Feinstein 2016), contribuyendo sobremanera al establecimiento del discurso de la culpa materna.

Durante el trabajo de campo ha emergido el concepto de madre nevera en la narrativa de las madres, a pesar de que según la bibliografía especializada se encontraba prácticamente extinguido. Este hecho demuestra el profundo calado que han tenido estas interpretaciones psicoanalíticas en la vida de las madres. Conviene recordar en este punto que se trata de madres de personas adultas con autismo y que sus vivencias en estos ámbitos se suceden desde unos veinte años atrás.

$\mathrm{Si}$, a mí no me ha pasado, pero yo he visto informes donde pone que si el niño tiene esos comportamientos es porque su madre no ha pasado el tiem-

6 Disponible en: <https://elpais.com/diario/1994/03/21/sociedad/764204424_850215.html>. Fecha de acceso: 11 jun. 2018.

7 La emergencia en el trabajo de campo del concepto «madre nevera» ha contribuido a que nos centremos en las madres de manera particular, ya que la práctica totalidad de las entrevistadas aluden a ese concepto y a cómo en algún momento de su periplo diagnóstico los profesionales médicos les refirieron esta cuestión como factor explicativo. Esta circunstancia ha supuesto una sorpresa por cuanto la literatura considera que dicha noción es obsoleta. 
po suficiente con él. A lo mejor en aquella época no había tanta información como hay ahora, pero hoy todavía hay corrientes de ese tipo, de hecho me contaron de una asociación de madres de Barcelona que la mayoría de las entidades en Cataluña se guían por la influencia francesa, del psicoanálisis, y todavía están con esto de las madres nevera. Decirle eso a una madre es brutal, porque de por sí ya el diagnóstico te hunde, si encima te dicen que es por tu culpa, pues imagínate (Carmen, madre de un chico con autismo de 19 años).

Los profesionales de la salud mental han definido el espacio de la normalidad y han generado la frontera a partir del establecimiento de criterios diagnósticos que no solo asignan una etiqueta a la persona autista, sino que deslocaliza a las madres respecto del ejercicio normativo de la maternidad. A través de estos discursos médicos se las sitúa en un espacio liminar en el que forzosamente ha de desarrollarse una maternidad diferente. Todo ello se materializa en una serie de discursos y prácticas que culpabilizan a las madres de las personas con autismo:

Ella (la psiquiatra) se centró en que yo le estaba exigiendo demasiado a la niña, que la tenía que dejar más a su ritmo... Llegó a decirme que si la niña necesitaba más tiempo que yo debía dejar de trabajar [...]. Planteaba que el autismo de mi hija era por entrar y salir de la guardería (Chari, madre de una chica con autismo de 27 años).

El afecto de estas madres hacia sus hijos e hijas se torna central a la hora de identificar qué elementos son definitorios en la aparición del autismo. Determinadas recomendaciones médicas como los besos y los abrazos inducen un tipo de maternidad que refleja más un deseo o una recomendación de los profesionales en base a un ideal de buena madre compartido en el imaginario colectivo:

El pediatra no hacía más que decirme «tú dale muchos besos y abrazos, que note que estas cerca, que eres cariñosa». Claro yo al principio no entendía bien por qué me decía esto, pero con el tiempo he sabido que era por eso del síndrome de la madre fría. Supongo que en ese momento se pensaba que influía (María, madre de una chica con autismo de 19 años).

En ambas situaciones -el proceso diagnóstico y la asistencia a servicios de salud mental- estas mujeres experimentan una relación de asimetría con unos profesionales sanitarios que son propietarios de los conocimientos expertos y que trasladan sobre ellas la responsabilidad de cumplir las expectativas asocia- das a la buena maternidad. Pero al mismo tiempo, diagnostican a sus hijos como autistas lo que genera una situación que dificulta y condiciona el propio desarrollo normativo de sus maternidades. Aquí se materializa la tensión normalidad/anormalidad a la que nos referimos en la introducción, definiendo un espacio liminar en el que los factores sociales y culturales se activan y vehiculan la experiencia de la maternidad en el autismo.

Estamos ante maternidades diferentes porque son definidas en base a las necesidades de los hijos e hijas y tienen muy en cuenta la caracterización que se realiza de su discapacidad: generalmente asociada al déficit, a la carencia de una normalidad normativa y homogeneizadora que anula singularidades y cualidades de las personas. El discurso sobre la discapacidad de estos niños y niñas genera un modelo de maternidad diferente y segregador en base a esas explicaciones y que además cuenta con sus propias expectativas. Un modelo ideal que se ejemplifica a través de metáforas, entendidas como herramientas que vehiculan significados (Berger y Luckmann 2001) y que explica de manera acertada Sousa (2011: 232) cuando se refiere a las madres guerreras. Esto es,

una madre que al escuchar que no hay esperanza para su hijo, en lugar de retroceder y llorar; derriba muros, se abre paso a través de obstáculos, sigue su intuición incluso cuando la gente le dice que está loca. Es una madre que cree en la esperanza. Una madre que cree en los milagros y es capaz de seguir adelante con fuerza y determinación, incluso cuando su pareja duda de ella y no le ofrece apoyo. Una madre que nunca se da por vencida, incluso cuando llega a callejones sin salida.

Madres que, sin embargo, no prescinden del anhelo de una maternidad normativa; y cuyo comportamiento (siguiendo la metáfora de la madre nevera) se argumenta como causa de la propia discapacidad de su hijo. Maternidad y discapacidad se enredan en un nudo recursivo de discursos (expertos y legos) y prácticas (sanitarias y familiares) que coloca más a la madre que al propio hijo autista (reconocido por diagnóstico como discapacitado) en un limbo que solo propicia la desorientación, la incertidumbre y el desconcierto sobre qué maternidad ejercer y cómo desarrollarla.

La psiquiatra decía que yo agobiaba a mi hija y que no le había prestado toda la atención que ella necesitaba. Decía que las mujeres en mi situación lo que tenían que hacer era dejar de trabajar para 
prestar atención a los hijos (Chari, madre de una chica con autismo de 27 años).

Con el uso de ambas metáforas, la madre nevera y la madre guerrera, se reproduce el discurso de la culpa materna en base a dos ideales de maternidad incompatibles entre sí pero que igualmente hace a las madres responsables del fenómeno del autismo:

\begin{abstract}
Mientras que antes se consideraba que las madres frías y cuidadoras autoritarias eran las responsables de causar la discapacidad intelectual de sus hijos, las madres guerreras son ahora responsables de curar la discapacidad, o al menos de acceder a una intervención que mitigue el impacto de la discapacidad en sus hijos. La implicación del arquetipo del héroe-guerrero es un cambio cultural en las expectativas de las madres de niños con discapacidad intelectual, aunque las imágenes actualizadas no alivian las cargas sociales asignadas a las madres de niños con discapacidad intelectual ni disminuyen la exposición de las madres a los sistemas de desigualdad (Sousa 2011: 221).
\end{abstract}

\section{CONSTRUYENDO MATERNIDADES EN TORNO AL AUTISMO}

Esta aproximación etnográfica nos ofrece una mirada valiosa para comprender las experiencias vividas por las madres de personas autistas. Tal como indica Nadesan (2005) la historia del autismo está inexorablemente unida a la historia de la maternidad, una maternidad ideal que es confrontada y re-creada con elementos como los saberes médicos expertos, el estigma de la no-normalidad y el discurso inculpatorio. Este último puede rastrearse en numerosa literatura médica y psiquiátrica, y se introduce en la disciplina psicológica manteniendo su vigencia durante largo tiempo (Waltz 2012); el argumento es bien simple: el desarrollo de los hijos se infiere básicamente del comportamiento de sus madres. Esta construcción de la culpa materna es aplicable en ambas direcciones, por exceso y por defecto de cariño, ya que madres muy cariñosas generan hijos dependientes emocionalmente, y las madres que crean distancia emocional pueden provocar autismo. De una u otra manera, las responsables para la comunidad científica siempre eran las madres:

El sentimiento de culpa que sienten padres y madres cuando reconocen públicamente la discapacidad de sus hijos e hijas es en parte resultado de actuaciones profesionales en relación a la idea de maternidad, unido a las tensiones con los servicios médicos y educativos, a pesar de los avances en la generación de teorías sobre funcionamiento familiar y solidaridad en el contexto de la discapacidad (Grinker 2008: 83).

Todos estos elementos forman parte de la cotidianidad de unas madres cuyos relatos construyen una identidad personal rescatada de las profesiones médicas, psicológicas, educativas y de asistencia social, en la que ellas dan un nuevo significado a la crianza de los niños y niñas con autismo (Sousa 2011). Es una estrategia que permite que estas protagonistas puedan situarse en el centro de la acción, y recuperar la propiedad del discurso en torno al autismo, sin que este sea considerado inferior por ser profano y por tanto no científico.

Yo fui a su pediatra varias veces y le dije que mi hijo no hablaba, tenía problemas de sueño... Y él me decía que bueno, que ya hablará. Y yo insistía porque yo le notaba algo, porque yo tenía otro hijo y entonces notas la diferencia. Y el médico me miraba como si estuviera loca o si fuera una exagerada (Loli, madre de un chico con autismo de 35 años).

Esta consideración tiene repercusiones sociales de gran calado a varios niveles. En primer lugar, es lógico pensar que con todas estas experiencias las madres y padres pusieran todo su empeño en encontrar respuestas a sus preguntas y atención a sus demandas. Esta inquietud se ha materializado en el conocido como movimiento social del autismo, una escisión del movimiento asociativo de la discapacidad, cuya motivación ha sido la búsqueda de servicios que presten atención especializada a las necesidades de sus hijos e hijas (Ryan y Runswick-Cole 2008; Waltz 2012).

\footnotetext{
Yo buscaba sobre todo que supieran tratar a mi hijo y que me pudieran enseñar a mí. Para mí eso era un apoyo fundamental para poder salir del duelo, de ese agobio que tiene uno cuando te diagnostican a un hijo con autismo. Yo siempre lo digo, para mí la asociación ha sido lo más, a mí me ha salvado la vida, psicológicamente hablando, porque estaba tan hundida y tan perdida en este tema que yo decía imadre mía como esta gente no me ayuden a salir de esta, no salgo! (Carmen, madre de un chico con autismo de 19 años).
}

Este movimiento asociativo que se inicia a partir de los años 80 del siglo XX, surge como indica Chamak (2008) en paralelo a un cambio profundo en la conceptualización del autismo que se refleja tanto en los modos de intervención profesional como en la orientación del propio movimiento asociativo: 
La emergencia de metodologías socioeducativas en Estados Unidos ha provocado una movilización a gran escala alrededor de los cambios en relación a la definición de autismo y sus intervenciones en muchos países. Estos factores contextuales han inducido una revisión en la manera en la que las asociaciones de padres y madres han evolucionado desde una posición de resignación y sumisión respecto a las decisiones de los psiquiatras, hacia una posición ofensiva que redefine el autismo no como una enfermedad psiquiátrica, sino una discapacidad que puede ser contrarrestada a través de métodos conductuales y educativos (Chamak 2008: 77).

Estas organizaciones, que se configuran como asociaciones de "afectados» al tiempo que como instituciones prestadoras de servicios especializados (tercer sector), ponen en funcionamiento metodologías novedosas, capaces de ofrecer un tipo de ayuda desconocida hasta entonces por las familias.

A través de la asociación [...] vino un terapeuta a casa, que trabajó mucho con nuestra hija, y la verdad es que ella lo pasó mal al principio, con las normas y las limitaciones, las agendas que ellos hacen y al final consiguió mucho de ella, era una nueva forma de trabajar, muy novedosa [...]. En la asociación tú escuchas a otras personas y te sientes más acompañado, sientes que te entienden... Sin decir nada tú ya sabes lo que está pasando, tú vas allí y ves a alguien con un arañón en la cara y ya sabes lo que ha pasado. Y bueno echas alguna lágrima y es que es así, te sientes comprendido [...] están siempre que los necesitamos (Chari y Antonio, padres de una chica con autismo de 27 años $^{8}$ ).

$\mathrm{Y}$ que han contribuido sobremanera en el afrontamiento de los problemas de sus hijos e hijas:

Si yo no hubiera tenido la asociación esto habría sido mucho más duro. Por eso yo amo tantísimo a la asociación: encuentras gente en la misma situación que tú, y los profesionales saben de autismo (Rosa, madre de un chico con autismo de 27 años).

8 Es este el único caso de los analizados durante el trabajo de campo en el que participa un padre de una persona con autismo. Existe, tal como se indica en la literatura (Bell 2004; Torres 2004; Ennis 2014; Fernández 2014; Kelly 2016; Gutiérrez y González 2017) y se ha evidenciado en el trabajo de campo, una delegación explícita sobre las madres a la hora de responsabilizarse del cuidado de los hijos e hijas con discapacidad: ellas pueden delegar en otros miembros de la familia tareas concretas, pero la responsabilidad y la coordinación de los cuidados sigue siendo suya. Esta responsabilidad sobre los cuidados se traslada también a la hora de contarlo. En el apartado sobre metodología profundizamos más en esta cuestión.
Al mismo tiempo, en el contexto de la asociación se produce un reconocimiento explícito del papel de las madres, de sus discursos y experiencias. Un elemento fundamental a este respecto es el cuestionamiento de esas explicaciones con forma de metáfora (madres frías, madres guerreras) que permite flexibilizar el peso identitario de la maternidad para las mujeres, al tiempo que amplía las opciones de maternidades posibles. No obstante, «el discurso inculpatorio permanece en un imaginario colectivo que ha desplazado a las madres desde la frialdad al heroísmo: ahora las madres son las salvadoras de sus hijos, y en definitiva deben procurar la normalidad por todos los medios posibles (dietas, colegios especiales, medicación, juguetes terapéuticos)» (Waltz 2015: 355). Las madres heroínas atacan ${ }^{9}$ los síntomas del autismo, desde una posición de lucha, de activismo. Socialmente deben hacerlo, esa parece su obligación. Se desarrolla, por tanto, una nueva presión social hacia la búsqueda de la normalidad en un contexto de escasos servicios públicos (estado neoliberal), lo cual no hace otra cosa que «abrir las puertas a la mercantilización de los servicios que estas personas necesitan» (Waltz 2015: 356). En cualquiera de sus configuraciones, el coste de este discurso de culpa materna o de obligación materna es muy elevado, y no solo a nivel económico, a nivel psicológico existe un riesgo grave para ellas que debe ser evidenciado ${ }^{10}$.

Analizadas etnográficamente, las experiencias de las madres de personas con autismo muestran con claridad la tensión entre normalidad y discapacidad. Al mismo tiempo, la evolución de los discursos y las prácticas implementadas por ellas parecen contribuir a encarar esa tensión de manera más satisfactoria y funcional. Este proceso difícilmente puede entenderse sin el concurso del movimiento asociativo, que además de buscar respuestas que no se hallaban en el contex-

9 Nos remitimos aquí a la metáfora de la guerra, utilizada con maestría por Susan Sontag en su análisis de la enfermedad (2003).

10 Tal como señala Waltz (2015) en la literatura científica se explicitan los discursos sobre la responsabilidad de las mujeres madres de personas con autismo. Dicha responsabilidad se ha ido derivando desde el discurso inculpatorio por la falta de afecto, hacia un nuevo papel como coordinadoras de cuidados, removedoras de obstáculos y agentes de consumo de productos y servicios dirigidos a sus hijos e hijas. El foco siempre se sitúa en las madres, siguiendo la lógica patriarcal inserta en la sociedad que todos y todas producimos. 
to médico, ha situado a las madres y a las familias como agentes activos en la construcción discursiva y práxica del autismo, contribuyendo a ensanchar las fronteras del conocimiento sobre el mismo y haciendo explícitas sus dimensiones socio-culturales.

\section{CONCLUSIONES}

Al retratar la tensión discursiva y práxica que experimentan las madres de personas con autismo accedemos empíricamente al espacio liminal en el que se encuentran la normalidad y la diversidad. Este espacio conflictivo tiene una doble vinculación: la caracterización de las personas con autismo y el ejercicio de la maternidad. Maternidad y autismo conforman un nudo tensional que ilustra de manera muy clara los imaginarios hegemónicos, los modelos ideales de maternidad y el sustrato desde el que se pretende la integración y normalización de la discapacidad en nuestra sociedad. Todo ello, si conseguimos aprehenderlo en su complejidad, nos permite reflexionar tanto sobre las dimensiones sociales y culturales del autismo como sobre las maternidades diferentes.

Nuestro estudio muestra que la antropología, a nivel teórico, y la etnografía, desde una perspectiva metodológica, conforman una estrategia especialmente indicada para desvelar cómo la normalidad es socialmente construida y personalmente vivida, configurando un espacio contestado, tanto en lo que respecta al autismo como a la propia maternidad. Especialmente interesante resulta dilucidar cómo la resolución de esa normalidad imposible, supone costes insuperables para las personas que quedan situadas en sus fronteras, sean madres o discapacitados, así como las estrategias que estos desarrollan para ganar un cierto espacio en la normalidad desde su anormalidad. Esta perspectiva de estudio tiene tres consecuencias analíticas convergentes, todas ellas vinculadas a la construcción de identidades - tanto discursivas como prácticas - de los sujetos que habitan el mundo del autismo y su maternidad.

Por un lado, reconocer la dimensión socio-cultural del autismo nos permite considerarlo desde bases analíticas más amplias a las convencionales, lo que facilita el reconocimiento de las personas con autismo como sujetos. Desde aquí es más fácil atender sus nuevas expresiones identitarias vinculadas, por ejemplo, a movimientos como el de neurodiversidad (Ortega 2009).
Asimismo, la articulación de la maternidad y la discapacidad, nos remite a una necesaria relativización del ejercicio materno. Nuestro recorrido etnográfico por distintas trayectorias y posicionamientos de mujeres madres de personas con autismo, ilustra su diversidad y cómo esta se confronta con modelos hegemónicos. Si bien esta circunstancia no es privativa del mundo de la discapacidad quizá en él toma una especial consistencia por su liminalidad, lo que permite ser extrapolado a otros ámbitos de maternidades más «normalizadas», en los que también se pugna por modelos más plurales y diversos.

Curiosamente, y a pesar de la desigual naturaleza de uno y otro proceso identitario (el de las personas con autismo y el de sus madres), ambos confluyen en la conformación de movimientos asociativos en torno a los cuales se consolidan cambios paradigmáticos en la consideración de la discapacidad y de la maternidad. Derechos de ciudadanía, emancipación, asistencialismos y modelos de madre forman parte de un gran campo discursivo y práxico que problematiza tanto el estatus de los discapacitados y sus madres como el del propio conocimiento científico, todo al mismo tiempo.

En definitiva, esta aproximación a las madres de personas con autismo desde una perspectiva antropológica, pone al descubierto tanto la oportunidad como la necesidad de encarar este tipo de fenómenos desde la propia experiencia de sus protagonistas. Una vez fijado este tipo de experiencias, deberán ponerse en diálogo con los relatos científicos y la intervención administrativa, referentes estos que con excesiva frecuencia han sido asumidos como únicos y exclusivos para comprender la realidad del autismo. Nuestro trabajo muestra cuanto camino queda aún por recorrer.

\section{BIBLIOGRAFÍA CITADA}

Aguado Díaz, Antonio León. 1995. Historia de las deficiencias. Madrid: Escuela Libre.

Allué, Marta. 2002. «El etnógrafo discapacitado. algunos apuntes sobre la observación de las conductas frente a la discapacidad». Acciones e Investigaciones Sociales, 15: 57-70.

Alvarado López, M.a Cruz, Susana Andrés del Campo y Rodrigo González Martín. 2007. «Discapacidad: estigma y concienciación». Comunicación e cidadanía: Revista Internacional de Xornalismo Social, 1: 203-222. 
APA American Psychiatric Association 2013. Diagnostic and Statistical Manual of Mental Disorders, 5th Edition. Washington DC: APA.

Araya Umaña, Sandra. 2007. «De lo invisible y lo cotidiano. Familias y discapacidad». Actualidades Investigativas en Educación, 7 (3): 1-21.

Asenjo Carbia, Alberto. 2011. Autismo y etnografía: interacciones sociales en un centro educativo y terapéutico. Trabajo Final de Máster. Universidad de Barcelona.

Bagatell, Nancy. 2010 «From cure to community: Transforming notions of autism». Ethos, 38(1): 33-55.

Beckett, Angharad E. 2015. "Anti-opressive pedagogy and disability: possibilities and challenges». Scandinavian Journal of Disability Research, 17: 76-94.

Bell, Susan E. 2004. «Intensive performances of mothering: a sociological perspective». Qualitative Research, 4(1): 45-75.

Berger, Peter L. y Thomas Luckmann. 2001. La construcción social de la realidad. Buenos Aires: Amorrortu Editores.

Bettelheim, Bruno. 2012. La fortaleza vacía: autismo infantil y el nacimiento del yo. Buenos Aires: Paidós.

Bourdieu, Pierre. 2003. El oficio de científico. Ciencia de la ciencia y reflexividad. Barcelona: Anagrama.

Canguilhem, Georges. 1970. Lo normal y lo patológico. Buenos Aires: Siglo Veintiuno Editores.

Chamak, Brigitte. 2008. «Autism and social movements: French parents' associations and international autistic individuals' organisations». Sociology of Health \& IIIness, 30(1): 76-96.

Cisneros, José Luis. 2010. «La percepción subjetiva de la discapacidad. Una mirada teórico-empírica». Veredas Especial Revista del Pensamiento Sociológico, 11: 159-181.

Comelles, Josep M. 2007. «Cultura, sociedad y psiquiatras en España». Frenia, VII.

Comelles, Josep M. y Ángel Martínez Hernáez. 1993. Enfermedad, cultura y sociedad. Un ensayo sobre las relaciones entre la antropología social y la medicina. Madrid: Eudema. Universidad Complutense de Madrid.

Corbett, Jenny. 1997. «Independent, proud and special: Celebrating our differences», en Len Barton y Mike Oliver, Disability Studies: Past, Present and Future: 90-98. Leeds: The Disability Press.

Daley, Tamara C. 2002. "The need for cross cultural research on the pervasive developmental disorders». Transcultural Psychiatry, 39 (4): 531-550.

De Miguel, Jesús M. 1980. «Introducción al campo de la Antropología Médica», en M. Kenny y J. M. De Miguel, La Antropología Médica en España: 11-40. Barcelona: Anagrama.

Dyches, Tina M., Lynn K. Wilder, Richard R. Sudweeks, Festus E. Obiakor y Bob Algozzine. 2004. "Multicultural issues in autism». Journal of Autism and Developmental Disorders, 34(2): 211-222.
Ennis, Linda Rose. 2014. Intensive Mothering: The Cultural Contradictions of Modern Motherhood. Toronto: Demeter Press.

Esteban, Mari Luz. 2000. «La maternidad como cultura. Algunas cuestiones sobre la lactancia materna y cuidado infantil», en Enrique Perdiguero y Josep M. Comelles (eds.), Medicina y cultura. Estudios entre la antropología y la medicina: 207-226. Barcelona: Edicions Bellaterra.

Feinstein, Adam. 2016. Historia del Autismo. Conversaciones con los pioneros. Ávila: Autismo Ávila.

Fernández Pujana, Irati. 2014. Feminismo y maternidad: ¿̇una relación incómoda? Vitoria-Gasteiz: Emakunde. Instituto Vasco de la Mujer.

Ferreira, Miguel A. V. 2007. «Prácticas sociales, identidad y estratificación: tres vértices de un hecho social, la discapacidad». Intersticios: Revista Sociológica de Pensamiento Crítico, 1(2): 1-15

Ferreira, Miguel A. V. y Carolina Ferrante. 2011. «Cuerpo y habitus: El marco estructural de la experiencia de la discapacidad». Intersticios: Revista Sociológica de Pensamiento Crítico, 5(2): 85-101.

Flyvbjerg, Bent. 2004. "Cinco malentendidos acerca de la investigación mediante los estudios de caso». Revista Española de Investigaciones Sociológicas (REIS), 106: 33-62.

Foucault, Michael. 2000. Los anormales. Argentina: Fondo de Cultura Económica.

Foucault, Michael. 2004. El nacimiento de la clínica: Una arqueología de la mirada médica. Argentina: Siglo XXI.

García Villamisar, Domingo, Javier Cabanyes, Araceli Del Pozo y Carmen Muela. 2006. Educación de personas con autismo. Madrid: Consejería de Educación. Comunidad de Madrid.

Goffman, Erving 2012. Estigma, la identidad deteriorada. Buenos Aires: Amorrortu editores.

Gómez Bernal, Vanessa. 2014. «Análisis de la discapacidad desde una mirada crítica: Las aportaciones de las teorías feministas». Estudios Pedagógicos, XL(2): 391-407.

Grinker, Roy Richard. 2008. Unstrange minds. A father remaps the world of autism. Cambridge: Icon Books.

Grinker, Roy Richard. 2010. «Commentary: On being autistic, and social». Ethos 38(1): 172-178.

Guber, Rosana. 2001. La etnografía. método, campo y reflexividad. Bogotá: Grupo editorial Norma.

Guerrero Muñoz, Joaquín. 2011. «Humanizando la discapacidad: De la etnografía al compromiso en la investigación sociocultural de la discapacidad intelectual». Revista de Antropología Experimental, 11: 127-138.

Gutiérrez González, Angélica y M. a Carmen González Camacho. 2017. «El trabajo de cuidar: una aproximación a los hombres cuidadores de personas con Alzheimer en la ciudad de Sevilla». Revista Iberoamericana de Relaciones Laborales, 35: 77-90.

Hays, Sharon. 1996. The cultural contradictions of motherhood. New Haven, CT: Yale University Press. 
Hollin, Gregory. 2014. "Constructing a social subject: Autism and human sociality in the 1980s». History of the Human Sciences, 27(4): 98-115.

Imaz Martínez, Elixabete. 2010. Mujeres gestantes, madres en gestación. Representaciones, modelos y experiencias en el tránsito a la maternidad de las mujeres vascas contemporáneas. Leioa: Universidad del País Vasco.

Jociles Rubio, María Isabel. 1999. «Las técnicas de investigación en antropología. Mirada antropológica y proceso etnográfico». Gazeta de Antropología, 15.

Kelly, Christine. 2016. Disability politics and care: the challenge of direct funding. Vancouver: UBC Press.

Kim, Hyun Uk. 2012. "Autism across cultures: rethinking autism», Disability \& Society, 27(4): 535-545. doi: <http://doi. org/10.1080/09687599.2012.659463>.

Malinowski, Bronislaw. 1986. Los argonautas del pacífico occidental. Barcelona: Planeta de Agostini.

Manouilenko, Irina y Susanne Bejerot. 2015. «Sukhareva: Prior to Asperger and Kanner». Nordic Journal of Psychiatry, Early Online: 1-4.

Menéndez, Eduardo L. 1990. Antropología médica. México: Centro de Investigaciones y Estudios Superiores en Antropología Social.

Menéndez, Eduardo L. 2018. Poder, estratificación social y salud. Colección Antropología Médica. Barcelona: Medical Anthropology Research Center. Publicaciones Universidad Rovira i Virgili.

Nadesan, Majia Holmer. 2005. Constructing Autism: Unravelling the 'Truth' and Understanding the Social. London: Routledge.

Oliver, Mike. 1998. «¿Una sociología de la discapacidad o una sociología discapacitada?», en Len Barton (ed.), Discapacidad y sociedad: 34-58. Madrid: Ediciones Morata.

Ortega, Francisco. 2009. "The cerebral subject and the challenge of neurodiversity». Biosocieties, 4: 425-445.

Osa Etxebeste, Joana. 2018. «La vida con Paul. Re-construyendo la experiencia con una hija o hijo con lesión cerebral». Bilbao: Universidad del País Vasco. Disponible en: https:// addi.ehu.es/handle/10810/24947

Palacios, Agustina. 2008. El modelo social de discapacidad: orígenes, caracterización y plasmación en la Convención Internacional sobre los derechos de las personas con discapacidad. Colección Cermi, 36. Madrid: Ediciones Cinca.

Palacios, Agustina y Javier Romañach Cabrero. 2008. «El modelo de la diversidad: Una nueva visión de la bioética desde la perspectiva de las personas con diversidad funcional (discapacidad)». Intersticios: Revista Sociológica de Pensamiento Crítico, 2(2): 37-47.
Ramos, María Alejandra. 2010. «¿Qué es el autismo? La experiencia de padres inmersos en la incertidumbre». Intersecciones en Antropología, 11(1): 73-88.

Rivera Amarillo, Claudia Patricia. 2004. Aprender a mirar: El discurso sobre el autismo. Bogotá: Encuentros. Universidad Nacional de Colombia.

Rodríguez Díaz, Susana y Miguel A. V. Ferreira. 2010. «Desde la dis-capacidad hacia la diversidad funcional. un ejercicio de dis-normalización». Revista Internacional de Sociología, 68(2): 289-309.

Rosato, Ana y María Alfonsina Angelino (coords.). 2009. Discapacidad e ideología de la normalidad. Desnaturalizar el déficit. Buenos Aires: Noveduc.

Ruiz Ballesteros, Esteban. 2013. «Hacia la operativización de la complejidad en ciencias sociales», en E. Ruiz Ballesteros y J. L. Solana Ruiz (eds.), Complejidad y Ciencias Sociales: 137172. Sevilla: Universidad Internacional de Andalucía.

Ryan, Sara y Katherine Runswick-Cole. 2008. «From Advocate to Activist? Mapping the Experiences of Children on the Autism Spectrum». Journal of Applied Research in Intellectual Disabilities, 22: 43-53.

Seoane Rodríguez, José Antonio. 2011. «¿Qué es una persona con discapacidad?» Ágora: Papeles de filosofía, 30(1): 143161.

Solomon, Olga. 2010. "Sense and the senses: Anthropology and the study of autism». Annual Review of Anthropology, 39: 241-259.

Sontag, Susan. 2003. La enfermedad y sus metáforas. El sida y sus metáforas. Madrid: Taurus.

Sousa, Amy C. 2011. «From refrigerator mother to warrior héroes: the cultural identity transformation of mothers raising children with intelectual disabilities». Symbolic Interaction, 34(2): 220-243.

Torres Dávila, María Soledad. 2004. Género y discapacidad: más allá del sentido de la maternidad diferente. Quito: Ediciones Abya-Yala.

Verdugo Alonso, Miguel Ángel. 2003. La concepción de la discapacidad en los modelos sociales, en Mesa Redonda ¿Qué significa la discapacidad hoy? Modelos conceptuales. Disponible en: <http://www.um.es/discatif/TEORIA/VerdugoModelosSoc.pdf>. Fecha de acceso: 4 abr. 2019.

Villa Fernández, Nuria y Soledad Arnau Ripollés. 2008. «Iniciativas de vida independiente en España: Pasos para alcanzar un derecho humano», en Actas del VI Congreso Internacional de Filosofía de la Educación "Educación, Conocimiento y Justicia». Madrid: Universidad Complutense de Madrid.

Waltz, Mitzi. 2012. "Images and narratives of autism within charity discourses». Disability \& Society, 27(2): 219-233.

Waltz, Mitzi. 2015. "Mothers and autism: the evolution of a discourse of blame». AMA, Journal of Ethics, 17(4): 353-358. 